PROCEEDINGS OF THE

AMERICAN MATHEMATICAL SOCIETY

Volume 129, Number 12, Pages 3589-3591

S 0002-9939(01)06181-0

Article electronically published on May 10, 2001

\title{
A SHORT PROOF OF ERGODICITY OF BABILLOT-LEDRAPPIER MEASURES
}

\author{
RITA SOLOMYAK
}

(Communicated by Michael Handel)

\begin{abstract}
Let $M$ be a compact manifold, and let $\phi_{t}$ be a transitive homologically full Anosov flow on $M$. Let $\widetilde{M}$ be a $\mathbb{Z}^{d}$-cover for $M$, and let $\widetilde{\phi_{t}}$ be the lift of $\phi_{t}$ to $\widetilde{M}$. Babillot and Ledrappier exhibited a family of measures on $\widetilde{M}$, which are invariant and ergodic with respect to the strong stable foliation of $\widetilde{\phi_{t}}$. We provide a new short proof of ergodicity.
\end{abstract}

Let $M$ be the unit tangent bundle of a compact Riemann surface, and let $\phi_{t}$ be the geodesic flow on $M$. Let $\widetilde{M}$ be a $\mathbb{Z}^{d}$-cover of $M$, and let $\widetilde{\phi_{t}}$ be the lift of $\phi_{t}$. Babillot and Ledrappier [BL96] constructed a family of measures $\left\{\mu_{v}\right\}_{v \in \mathbb{R}^{d}}$ on $\widetilde{M}$, which are preserved by the horocycle flow on $\widetilde{M}$, and proved that they are ergodic. Kaimanovich K98 gave an alternative proof of ergodicity in case of Liouville measure $\left(=\mu_{0}\right)$.

The construction of Babillot and Ledrappier generalizes to a $\mathbb{Z}^{d}$-extension of an arbitrary Anosov flow, where it gives a family of measures $\left\{\mu_{v}\right\}_{v \in \mathbb{R}^{d}}$ invariant w.r.t. the strong stable foliation for $\widetilde{\phi_{t}}$. The lift of the measure of maximal entropy coincides with $\mu_{0}$. In this setting Pollicott P98] proved ergodicity of $\mu_{0}$ by a different method, and Coudene [99] obtained a generalization to all $\left\{\mu_{v}\right\}_{v \in \mathbb{R}^{d}}$. We give a new proof of ergodicity of $\mu_{v}$ for all $v \in \mathbb{R}^{d}$ using a theorem of Guivarc'h [G89.

The flow $\phi_{t}: M \rightarrow M$ is said to be homologically full if there exists a closed orbit of $\phi_{t}$ in each homology class; see [S93].

Theorem (Babillot and Ledrappier BL96], Pollicott [P98, Coudene [C99]). Let $\left(\widetilde{M}, \widetilde{\phi}_{t}\right)$ be a $\mathbb{Z}^{d}$-extension of a transitive Anosov flow, and let $\left\{\mu_{v}\right\}_{v \in \mathbb{R}^{d}}$ be the family of Babillot-Ledrappier measures on $\widetilde{M}$. If $\phi_{t}$ is homologically full, then for any $v \in \mathbb{R}^{d}$ the strong stable foliation of $\widetilde{\phi}_{t}$ is ergodic w.r.t. $\mu_{v}$.

Proof. Using a method of [BM77], the problem can be reformulated in terms of symbolic dynamics; see [P98]. Let $(\Sigma, T)$ be a topologically mixing one-sided shift of finite type (see e.g. [B75] for the definitions). Let $r: \Sigma \rightarrow \mathbb{R}^{+}$and $\psi: \Sigma \rightarrow \mathbb{Z}^{d}$ be Hölder continuous functions, and assume that $\psi$ depends only on the first two coordinates, i.e. $\psi(x)=\psi\left(x_{1}, x_{2}\right)$. Consider the set

$$
\Lambda=\{(x, t, i): 0 \leq t \leq r(x)\} \subset \Sigma \times \mathbb{R}^{+} \times \mathbb{Z}^{d}
$$

with identifications $(x, r(x), j)=(T x, 0, j+\psi(x))$.

Received by the editors April 14, 2000.

2000 Mathematics Subject Classification. Primary 37A20.

(C)2001 American Mathematical Society 
Define an equivalence relation $R_{\Lambda} \subset \Lambda \times \Lambda$ by setting $(x, t, i) \sim(y, s, j)$ if there exist $m, n \geq 0$ such that

$$
\begin{aligned}
T^{m}(x) & =T^{n}(y), \\
r_{m}(x)-t & =r_{n}(y)-s, \\
i+\psi_{m}(x) & =j+\psi_{n}(y),
\end{aligned}
$$

where $r_{n}$ and $\psi_{n}$ denote the corresponding ergodic sums: $r_{n}(x)=r(x)+r(T x)+$ $\ldots+r\left(T^{n-1} x\right)$ and $\psi_{n}(x)=\psi(x)+\psi(T x)+\ldots+\psi\left(T^{n-1} x\right)$.

For any $v \in \mathbb{R}^{d}$, consider the potential $\phi_{v}: \Sigma \rightarrow \mathbb{R}$, given by $\phi_{v}=\lambda_{v} r(x)-$ $\langle\psi(x) \mid v\rangle$, where $\langle\cdot \mid \cdot\rangle$ stands for the scalar product and $\lambda_{v}$ is the unique real number such that topological pressure $P\left(\phi_{v}\right)=0$. Let $\nu_{v}$ be the eigenmeasure for the corresponding Perron-Frobenius-Ruelle operator (the reader is referred to e.g. B75] for the definitions). Set

$$
\mu_{v}=\nu_{v} \times e^{-\lambda t} d t \times e^{\langle z \mid v\rangle} d z
$$

Ergodicity of the strong stable foliation w.r.t. Babillot-Ledrappier measures amounts to ergodicity of $R_{\Lambda}$ w.r.t. $\mu_{v}$; see [BL96].

It is convenient to consider the space $Y=\Sigma \times \mathbb{R} \times \mathbb{Z}^{d}$ rather than $\Lambda$, where the equivalence relation $R_{Y}$ and measures $\mu_{v}$ are defined by (1)-(3) and (4), respectively. It suffices to prove ergodicity of $R_{Y}$ w.r.t. $m_{v}$, or, equivalently, w.r.t. $\nu_{v} \times d t \times d z$, where $d z$ is Haar measure on $\mathbb{Z}^{d}$.

Recall that a Hölder continuous function $f: \Sigma \rightarrow G$, where $G$ is a locally compact abelian polish group, is called periodic (see G89]) if there exist a nonconstant measurable $g: \Sigma \rightarrow \mathbb{S}^{1}$, a constant $z \in \mathbb{S}^{1}$ and a character $\gamma \in \hat{G}$ such that $\gamma \circ f(x)=z g(T x) \bar{g}(x)$. If $f$ is not periodic, it is called aperiodic. We set $G=\mathbb{R} \times \mathbb{Z}^{d}$ and $f(x)=(-r(x), \psi(x))$.

Lemma. If $\phi_{t}$ is homologically full, then $f$ is aperiodic.

(The proof is given below.)

Consider the skew-product transformation $T_{f}: \Sigma \times G \rightarrow \Sigma \times G$ defined by

$$
T_{f}(x, y)=(T x, y+f(x)) \text {. }
$$

By a theorem of Guivarc'h G89] (see also [AD99]), $T_{f}$ is exact w.r.t. $p \times m$, where $p$ is any Gibbs measure and $m$ is Haar measure on $G$. Now, let $R^{\prime}$ be the tail equivalence relation for $T_{f}$, i.e. $(x, y) \sim\left(x^{\prime}, y^{\prime}\right)$ iff for some $n$

$$
\begin{aligned}
T^{n}(x) & =T^{n}\left(x^{\prime}\right), \\
y+f_{n}(x) & =y^{\prime}+f_{n}\left(x^{\prime}\right) .
\end{aligned}
$$

Clearly, ergodicity of $R^{\prime}$ amounts to the exactness of $T_{f}$, so $R^{\prime}$ is ergodic. But $R^{\prime}$ is smaller than $R_{Y}$, so $R_{Y}$ is ergodic.

Proof of the Lemma. If $f$ is periodic, then there exist $\gamma \in \hat{G}$ and $z \in \mathbb{S}^{1}$ such that $\gamma \circ f_{n}(x)=z^{n}$ for any periodic $x \in \Sigma$ with period $n$. Fix $\alpha \in \mathbb{Z}^{d}$ and $\delta>0$. By a result of Sharp [S93], the number of periodic $x$ with $\psi_{n}(x)=\alpha$ and $r_{n}(x) \in(T-\delta, T]$, where $n$ is the period of $x$, is exponential in $T$. Since the roof function $r$ is strictly positive, the number of possible periods $n$ is linear in $T$. So, one can find $x, y$ with the same period $n$, such that

$$
0<\left|r_{n}(x)-r_{n}(y)\right|<2 \delta, \quad \psi_{n}(x)=\psi_{n}(y)=\alpha
$$


This shows that $\gamma(\cdot, \alpha)$ is constant in the first coordinate. Similarly, for any $\alpha, \beta \in$ $\mathbb{Z}^{d}$ one can find $x, y$ of the same period $n$ with $\psi_{n}(x)=\alpha, \psi_{n}(y)=\beta$, which shows that $\gamma$ is constant. Thus, $f$ is aperiodic.

Remark. The theorem of Guivarc'h [G89] was applied in the same way in [ANSS].

\section{REFERENCES}

[AD99] J. Aaronson and M. Denker. On exact group extensions. Preprint (1999) .

[ANSS] J. Aaronson, H. Nakada, O. Sarig, R. Solomyak. Invariant measures and asymptotics for some skew products. Preprint (1999).

[BL96] M. Babillot, F. Ledrappier, Geodesic paths and horocycle flow on abelian covers. in: Lie groups and ergodic theory (Mumbai, 1996) 1-32, Tata Inst. Fund. Res. Stud. Math. 14, Tata Inst. Fund. Res., Bombay, 1998. MR 2000e:37029

[B75] R. Bowen. Equilibrium states and the ergodic theory of Anosov diffeomorphisms, Lecture notes in Mathematics 470, Springer (1975). MR 56:1364

[BM77] R. Bowen, B. Marcus. Unique ergodicity for horocycle foliations, Israel J. Math. 13, (1977), 43-67. MR 56:9594

[C99] Y. Coudene, Cocycles and stable foliations of Axiom A flow. Preprint (1999).

[G89] Y. Guivarc'h. Proprietes ergodiques, en mesure infinie, de certains systemes dynamiques fibres. Ergod. Th. \&S Dynam. Sys. (1989), 9, 433 -453. MR 91b:58190

[K98] V. Kaimanovich, Ergodic properties of the horocycle flow. Preprint (1998).

[P98] M. Pollicott, $\mathbb{Z}^{d}$-covers of horosphere foliations, Discrete and Continuous Dynamical Systems (2000), Vol 6, 1, 147-154. CMP 2000:08

[S93] R. Sharp, Closed orbits in homology classes for Anosov flows, Ergod. Th. and Dyn. Sys. 13 (1993), 387-408. MR 94g:58169

Department of Mathematics, University of Washington, Box 35450, Seattle, WashINGTON 98195

E-mail address: rsolom@math.washington.edu 